\title{
Optimal Duration of Prophylactic Antibiotics Administration in Pancreaticoduodenectomy
}

\author{
Tomohisa Yamamoto, Sohei Satoi, Hiroaki Yanagimoto, So Yamaki, Satoshi Hirooka, Taku Michiura, \\ Kentaro Inoue, Yoichi Matsui
}

Corresponding author:

Sohei SATOI M.D.

Department of Surgery, Kansai

Medical University, 2-3-1, Shin-machi

Hirakata, 573-1191, Japan

Tel: +81-72-804-0101

Fax: $+81-72-804-0170$

E-mail: satoi@hirakata.kmu.ac.jp

Received: 05.12.2017

Accepted: 16.12 .2017

Copyright () Celsius Publishing House www.sgo-iasgo.com
Department of Surgery, Kansai Medical University, Osaka, Japan

\section{ABSTRACT}

Purpose: Pancreaticoduodenectomy (PD) is considered a complex surgical procedure that is associated with a high morbidity. There has been no report on the optimal duration of prophylactic antibiotics administration to date. Mortality and morbidity, including surgical site infection (SSI) after PD, were compared retrospectively taking into account the period of prophylactic antibiotics administration.

Methods: This study involved 158 patients who underwent PD between April 2006 and December 2010. Until March 2008 our hospital administered prophylactic antibiotics until post-operative day-2, but beginning in April 2008 they were administered only until postoperative day-1. Group A consisted of 71 consecutively treated patients who received cefmetazole (CMZ) as a prophylactic antibiotic intra-operatively and for 2 days postoperatively. Group B consisted of 87 consecutively treated patients who received CMZ intraoperatively and for 1 day post-operatively. During the study period, peri-operative management was standardized according to our departmental guidelines.

Results: There were no significant differences in post-operative complications between the two groups. The most commonly isolated microorganisms from surgical drainage fluid were Enterobacter and Enterococcus species. Pre-operative biliary drainage was an independent risk factor for SSI $(p<0.05)$.

Conclusion: Limiting prophylactic antibiotics administration until post-operative day-1 did not increase morbidity after PD. However, we recommend long-term administration of prophylactic antibiotics for patients who undergo pre-operative biliary drainage.

Key words: Prophylactic antibiotics, surgical site infection, Pancreaticoduodenectomy, pre-operative biliary drainage

\section{INTRODUCTION}

Pancreaticoduodenectomy (PD) is considered a complex surgical procedure that is associated with a high morbidity. High volume centers have reported that mortality after PD decreased less than $5 \%$, although post-operative morbidity was approximately $40 \%$, including postoperative pancreatic fistula (POPF), surgical site infections (SSI), intra-abdominal abscess, and delayed gastric emptying (DGE) (1-5). We have also reported that postoperative morbidity after PD 
decreased following introduction of new guidelines such as the modified Kakita method of pancreaticojejunostomy, early removal of closed-suction drains, and restricted use of pancreatic duct stenting (6).

Prophylactic use of antibiotics is recommended to prevent a variety of postoperative infections $(7,8)$. According to the Centers for Disease Control (CDC) guidelines for the prevention of SSI, antimicrobial prophylaxis should be administered to carry out clean operations (9). Administration of antimicrobial prophylaxis within 30 minutes of the first surgical incision, with intraoperative supplemental administration every 3 or 4 hours and postoperative administration for 24 hours or less, are the recommended timings and durations for the administration of antimicrobial prophylaxis in general surgery. Recently, a phase III, open-label, randomized controlled trial from Japan demonstrated non-inferiority of intraoperative antimicrobial prophylaxis after gastric cancer surgery, relative to extended antimicrobial prophylaxis (10). However, there has been no report on the optimal duration of antimicrobial prophylaxis for PD.

We previously followed conventional guidelines by administering prophylactic antibiotics until postoperative day- 2 in patients who underwent PD. However, since April 2008 we have adopted CDC guidelines and limited the duration of antibiotics administration until post-operative day 1 . Herein, we retrospectively compare mortality and morbidity, including SSI after $\mathrm{PD}$, taking into account the duration of prophylactic antibiotics administration.

\section{PATIENTS AND METHODS}

This study involved consecutively treated 158 patients who underwent PD including pylorus-preserving PD between April 2006 and December 2010. Group A consisted of 71 patients who underwent PD from April 2006 to March 2008. Patients in group A received cefmetazole (CMZ) as a prophylactic antibiotic $s$ intraoperatively and for 2 days post-operatively. Group B consisted of 87 patients who underwent PD from April 2008 to December 2010. Patients in group B received cefmetazole (CMZ) intra-operatively and for 1 day postoperatively.

Pancreaticojejunostomy (PJ) was performed using duct-to-mucosa anastomosis, as previously reported (11). We have carried out PJ without external or internal stenting of the pancreatic and biliary duct for patients since 2006. Peri-operative management was in accordance with departmental guidelines and clinical pathways (12). Briefly, on post-operative day (POD) 1 , the naso-gastric tube was removed if the drained fluid volume was less than $200 \mathrm{ml}$. We used closed-suctioned drains, and peri-pancreatic drainage fluid was collected and the amylase level measured and monitored on POD 3 and 6 and every 3 days thereafter as needed. The drainage tubes were removed from patients without infection-induced systemic inflammatory response syndrome (SIRS) (13) or POPF as defined by the International Study Group on Pancreatic Fistula (ISGPF) (14). For patients with POPF who were clinically diagnosed as having infection-induced SIRS, the perianastomotic drains were replaced on post-operative day 6 . For patients with continuous signs of infection, closed lavage with natural saline was performed to treat the infection focus and dilute the pancreatic juice, according to the departmental criteria for drain removal. On POD 3, patients were allowed to ingest a clear liquid diet, and enteral feeding was started if a tube was inserted. Discharge of patients from hospital was targeted for POD 14, but the actual discharge date depended on the following criteria: the patient could eat at least half of a meal, was afebrile, and had CRP levels that had decreased over time to less than 5 $\mathrm{mg} / \mathrm{dl}$. No patients received either prophylactic octreotide to prevent POPF development or thromboembolic prophylaxis with low molecular weight heparin.

All complications were recorded into a prospective pancreatic database. Prophylactic antibiotics were administered with a drip infusion before the initial skin incision as well as every 3 hours during the operation. Until POD 1 or POD 2, prophylactic antibiotics were administered every morning and evening. On the diagnosis of clinical sepsis (infection-induced SIRS) for 2 consecutive days, an experimental broad-spectrum antibiotic (carbapenem) was administered at least for 4 days, and exchange of drains or percutaneous drainage was performed as needed.

\section{Definition of postoperative complications}

The overall general and surgery-related complications were recorded in the patient's original clinical records. These included POPF, DGE, wound dehiscence, intra-abdominal infection, abdominal abscess formation, cardiopulmonary disorder, hemorrhagic complication, and other complications. Each day that a patient demonstrated clinical symptoms of SIRS was recorded (13). Clinical sepsis was considered to be present if a patient had any complication involving clinical symptoms of infection-induced SIRS that continued for more than two inpatient days. Clinical symptoms of 
SIRS within the first four post-operative days were excluded as a systemic response to surgical stress. POPF was defined according to ISGPF criteria: amylase level in surgical drainage fluid greater than three times the serum amylase activity on or after POD 3 (14). Intra-abdominal abscess was defined as a collection of pus or infected fluid that was confirmed by ultrasound, computed tomographic guided aspiration and culture. Intra-abdominal infection was regarded as microbiological findings of bacteria from surgical drainage fluid with clinical symptoms of sepsis. DGE was defined according to the ISGPS criteria (15). Wound dehiscence was diagnosed as an open wound regardless of the clinical presence of pus or microbiological findings confirming the presence of bacteria. According to CDC guidelines, development of SSI was also evaluated. Patients were classified according to the World Health Organization (WHO) BMI criteria, with patients having a BMI of 25 or more defined as overweight (16). Death of a patient for any reason was regarded as an in-hospital death.

\section{Microbiological culture in patients with clinical sepsis}

Samples of effusion fluid from wound dehiscence for bacterial culture examination were obtained from 27 patients. Samples of drained fluid were also submitted for bacterial culture examination from patients showing SIRS who had an intra-abdominal infection. Thirty-one patients who presented with clinical sepsis after drain removal had fluid collection in the abdominal space (intra-abdominal abscess) detected by imaging studies. The abscesses of these patients were punctured immediately and the drainage fluid submitted for bacterial culture examination.

\section{Statistical analysis}

Statistical analyses were performed using the JMP statistical program version 5.1 (SAS Inc. North Carolina, USA). All data are expressed as median values and ranges. The chi-square test or Fisher exact test was used for comparison of categorical variables, when appropriate. The Mann-Whitney U test was used for comparison of continuous variables. Multivariate analysis using logistic regression analysis was carried out to determine independent significant factors for postoperative complications following PD. P values less than 0.05 were considered significant.

\section{RESULTS}

\section{Comparison of clinical characteristics between the two groups}

Table 1 shows baseline and operative characteristics of the 158 patients enrolled in the study. None of these variables differed significantly between the two groups.

Table 1 - Patient Characteristics

\begin{tabular}{|c|c|c|c|}
\hline Parameters & Group $A(n=71)$ & Group B $(n=87)$ & $\mathrm{p}$ value \\
\hline Age (years old) & $67(39-90)$ & $66(33-82)$ & 0.133 \\
\hline Male:female & $50: 21$ & $53: 34$ & 0.211 \\
\hline Body mass index & $20.4(13.7-27.9)$ & $20.8(13.2-33.3)$ & 0.917 \\
\hline Disease (P:B:V) & $40: 13: 18$ & $53: 11: 23$ & 0.613 \\
\hline Benign:malignant ratio & $11: 60$ & $11: 76$ & 0.608 \\
\hline $\begin{array}{l}\text { Co-morbid disease }(-:+) \\
0.469\end{array}$ & $23: 48$ & & $33: 54$ \\
\hline Albumin $(\mathrm{g} / \mathrm{dl})$ & $3.7(1.9-4.7)$ & $3.8(1.9-4.8)$ & 0.515 \\
\hline Diabetes Mellitus $(-:+)$ & $49: 22$ & $67: 20$ & 0.259 \\
\hline Jaundice $(-:+)$ & $26: 45$ & $36: 51$ & 0.542 \\
\hline $\begin{array}{l}\text { Pre-operative biliary drainage }(-:+) \\
0.919\end{array}$ & $28: 43$ & & $35: 52$ \\
\hline Type of op (PD:PpPD) & $41: 30$ & $41: 46$ & 0.183 \\
\hline Operation time (min) & $479(275-714)$ & $449(259-711)$ & 0.304 \\
\hline Blood loss (ml) & $1047(272-3417)$ & $882(110-5238)$ & 0.272 \\
\hline Resection of other organs $(-:+)$ & $57: 14$ & $59: 28$ & 0.075 \\
\hline
\end{tabular}

The data are expressed as median and range.

$P: B: V$, pancreatic disease: biliary disease: ampulla of Vater disease; PD, pancreaticoduodenectomy;

$\mathrm{PpPD}$, pylorus preserving pancreaticoduodenectomy. 


\section{Comparison of post-operative complications}

As shown in Table 2, the overall post-operative complication rates were $43 \%$ for group A and $54 \%$ for group $\mathrm{B}$, respectively. There were no significant differences in frequencies of POPF, SSI, DGE, reoperation, or in-hospital deaths between the two groups. Moreover, no significant differences in duration of in-hospital stay (group A: 14(9-82) days, group B: 12 (8-101) days, $p=0.302$ ) or postoperative date of drain removal (group A: POD 6(3-50), group B: POD 3(3-52), $p=0.519$ ) were found between the two groups.

Remedial antibiotics were administered to patients with clinical sepsis (infection-induced SIRS) that continued for more than two consecutive days. Thirty-two patients in group A (31\%), and 31 patients in group B (36\%) received this therapy. The specific causes of their SIRS were POPF grade $B / C(n=9)$, Organ/Space SSI $(n=13)$, cholangitis $(n=3)$, pneumonia $(n=4)$, and others $(n=3)$ in group $A$, and POPF grade $B / C(n=14)$, Organ/Space SSI $(n=16)$, cholangitis $(n=7)$, pneumonia $(n=1)$, and others $(n=7)$ in group $B$. There were no significant differences between these clinical characteristics in groups $A$ and $B$. Moreover, there was no difference in the number of days until remedial antibiotics therapy was initiated, as shown in table 2. Carbapenem antibiotics were used most often for remedial antibiotics for both groups.

\section{Species of microorganisms detected by culture in surgical drainage fluid or ascites}

Waste fluid from 31 patients with SIRS who had an intra-abdominal infection or abscess was submitted for bacterial culture examination. A large variety of microorganisms was identified in surgically drained fluid or ascites cultures obtained after PD. The most commonly isolated microorganisms were Enterobacter species (34\% in group A and $27 \%$ in group B, respectively) and Enterococcus species (20\% and $27 \%$ ) as shown in fig. 1. The incidence of the drug-resistent microorganism (methicillin-resistant Staphylococcus aureus: MRSA and methicillin-resistant Staphylococcus epidermidis: MRSE) was one case in group $A$ and seven cases in group $B$, but this difference did not achieve statistical significance $(p=0.076)$.

\section{Species of microorganisms detected by culture in effusion from wound dehiscence}

Wound dehiscence was found in 9 patients in group $A$ and 18 patients in group $B$, respectively. Eight of the nine patients (89\%) in group $A$ and 14 of the 18 patients (78\%) in group B had positive effusion cultures. The most commonly isolated microorganisms were Enterobacter species (37\% in group A and $29 \%$ in group B, respectively) and Enterococcus species (18\% and $34 \%$ ) (fig. 2). A large variety of microorganisms was identified in effusions from wound dehiscence cultures. MRSA was found in one patient in group $B$ and none in group $A$, and this difference was not statistically significant.

\section{Analyses for SSI}

As shown in table 3, univariate analysis showed that

Table 2 - Comparison of post-operative complications

\begin{tabular}{lccc}
\hline Parameters & Group A $(\mathbf{n}=\mathbf{7 1})$ & Group B (n=87) & p value \\
\hline overall complications & $32 / 71(45 \%)$ & $47 / 87(50 \%)$ & 0.345 \\
\hline re-operation & $1 / 71(1 \%)$ & $1 / 87(1 \%)$ & 0.885 \\
\hline in-hospital death & $2 / 71(3 \%)$ & $2 / 87(2 \%)$ & 0.837 \\
\hline pancreatic fistula & $28 / 71(39 \%)$ & $32 / 87(37 \%)$ & $11 / 87(13 \%)$ \\
\hline Grade B/C & $10 / 71(14 \%)$ & $9 / 87(10 \%)$ & 0.732 \\
\hline DGE & $6 / 71(8 \%)$ & $0 / 87(0 \%)$ & 0.791 \\
\hline Hemorrhage & $0 / 71(0 \%)$ & $25 / 87(29 \%)$ & 1.000 \\
\hline SSI & $15 / 71(21 \%)$ & $18 / 87(16 \%)$ & 0.274 \\
\hline Organ/space SSI & $13 / 71(18 \%)$ & $18 / 87(21 \%)$ & 0.708 \\
\hline wound dehiscence & $9 / 71(13 \%)$ & $14 / 87(16 \%)$ & 0.183 \\
\hline wound infection & $8 / 71(11 \%)$ & $3 / 87(3 \%)$ & 0.384 \\
\hline Pneumonia & $4 / 71(6 \%)$ & $31 / 87(36 \%)$ & 0.296 \\
\hline Use of remedial antibiotics & $22 / 71(31 \%)$ & $8(2-20)$ & 0.532 \\
\hline Post-operative date initiated remedial antibiotics & $7(3-37)$ & $16 / 9 / 6$ & 0.625 \\
\hline Type of remedial Antibiotics (CA/NQ/CE) & $13 / 5 / 4$ & 0.845 \\
\hline
\end{tabular}

Table shows number of patients. Parentheses represent percentages of patients

DGE, delayed gastric emptying; SSI, surgical site infection ; CA,Carbapenem; NQ,New Quinolone; CE,cephem 

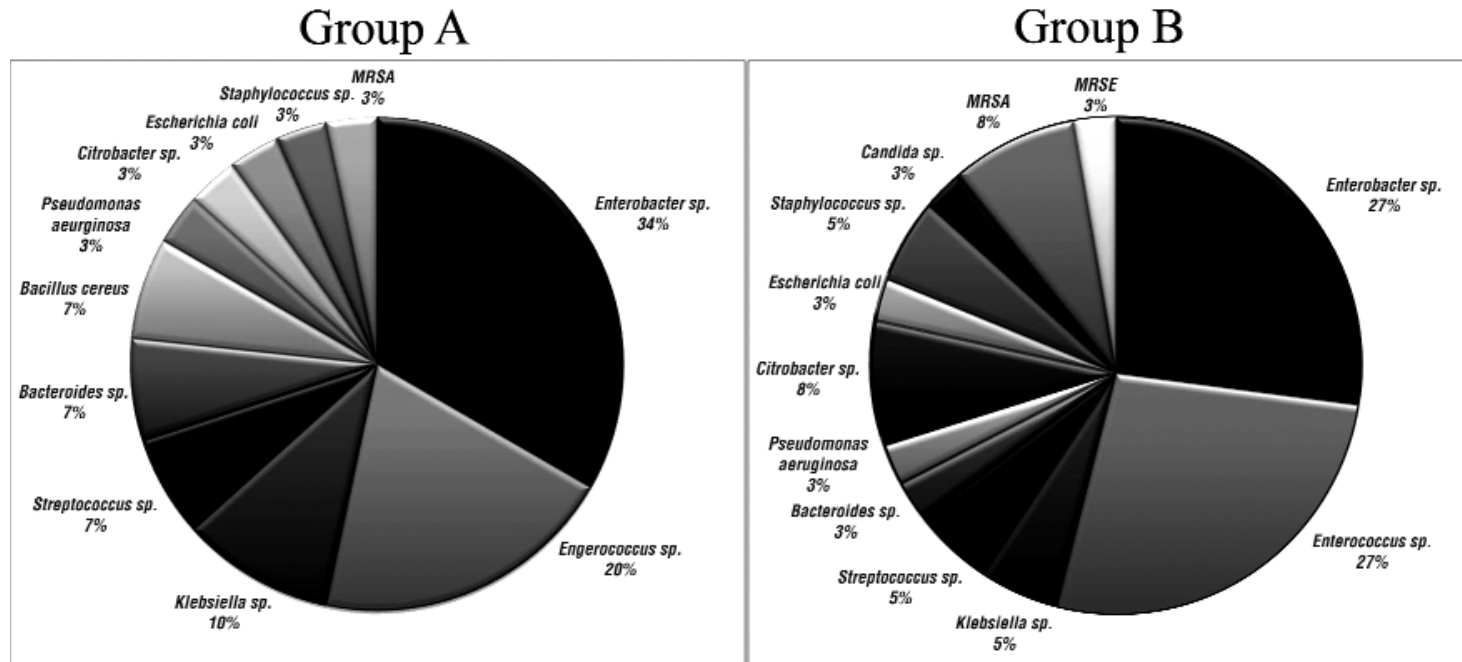

Figure 1 - Species of bacteria that were detected in surgical drainage fluid or ascites culture from 31 patients who had intra-abdominal infection of abscess. MRSA - methicillin-resistant Staphylococcus aureus; MRSE - methicillin-resistant Staphylococcus epidermidis. Bacteria were detected in 12 patients in Group A, and 21 patients in Group B

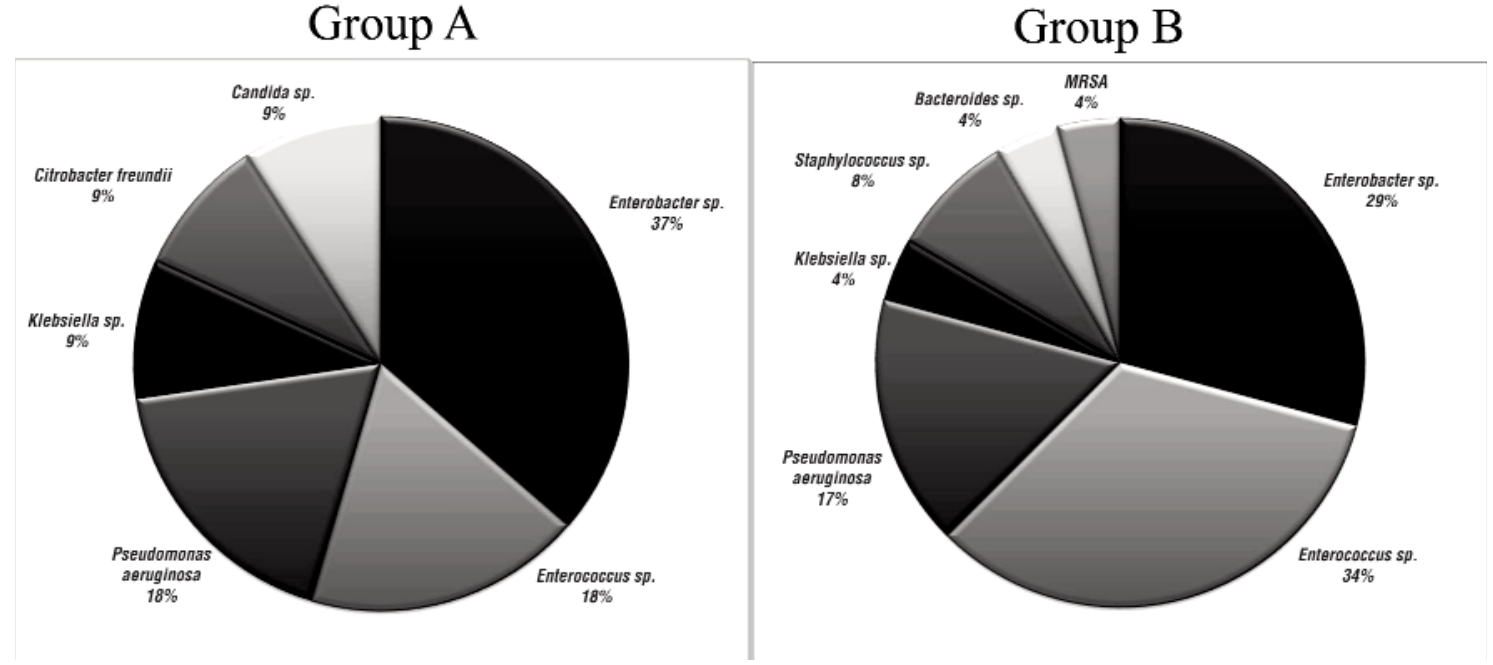

Figure 2 - Species of bacteria that were detected in effusions from wound dehiscence culture in 27 patients MRSA - methicillin-resistant Staphylococcus aureus; MRSE - methicillin-resistant Staphylococcus epidermidis. Bacteria were detected in 8 patients in Group A, and 19 patients in Group B.

a significant independent factor was whether the patient underwent preoperative biliary drainage (PBD) for superficial SSI. However, there were no significant independent factors affecting the overall SSI or the Organ/Space SSI incidences. Duration of prophylactic antibiotics administration did not affect the development of SSI, superficial SSI or Organ/Space SSI.

\section{DISCUSSION}

Antibiotic prophylaxis has proven effective in preventing post-operative infectious complications. The
1999 CDC guidelines defined surgical antibiotic prophylaxis as the very brief administration of an antimicrobial agent immediately prior to an operation (9). These guidelines recommended that antibiotic prophylaxis should target the most likely intra-operative contaminants and that, by the time the skin is incised, a bactericidal concentration of the drug should be established in serum and tissues. Actually, according to the result of a Questionnaire on Perioperative Antibiotic Therapy in Japan reported in 2003 (17), CEZ was administered to $14.9 \%$ and second-generation cephems were administered to $76.9 \%$ of patients undergoing PD at the 
Table 3 - Univariate analysis for SSI, Superficial SSI and Organ/space SSI

\begin{tabular}{|c|c|c|c|c|c|c|c|c|}
\hline \multirow[t]{2}{*}{ Category } & \multirow[t]{2}{*}{ Risk factor } & \multirow[t]{2}{*}{$n$} & \multicolumn{2}{|c|}{ Overall SSI } & \multicolumn{2}{|c|}{ Superficial SSI } & \multicolumn{2}{|c|}{ Organ/Space SSI } \\
\hline & & & $P$ value & $\mathrm{HR}(95 \% \mathrm{Cl})$ & $P$ value & $\mathrm{HR}(95 \% \mathrm{Cl})$ & $P$ value & $\mathrm{HR}(95 \% \mathrm{Cl})$ \\
\hline \multirow[t]{2}{*}{ BMI } & $25<$ & 141 & & 1 & & 1 & & 1 \\
\hline & $\geq 25$ & 17 & 0.128 & $2.29(0.78-6.45)$ & 0.258 & $2.10(0.54-6.72)$ & 0.826 & $0.86(0.19-2.88)$ \\
\hline \multirow[t]{2}{*}{ DM } & - & 116 & & 1 & & 1 & & 1 \\
\hline & + & 42 & 0.498 & $0.75(0.31-1.69)$ & 0.276 & $1.71(0.64-4.37)$ & 0.913 & $0.95(0.37-2.26)$ \\
\hline \multirow[t]{2}{*}{ Alb } & $\geq 3.8 \mathrm{~g} / \mathrm{dl}$ & 87 & & 1 & & 1 & & 1 \\
\hline & $3.8 \mathrm{~g} / \mathrm{dl}<$ & 71 & 0.467 & $1.31(0.63-2.92)$ & 0.958 & $0.98(0.39-2.46)$ & 0.109 & $1.94(0.86-4.61)$ \\
\hline \multirow[t]{2}{*}{ Disease } & Panc & 93 & & 1 & & 1 & & 1 \\
\hline & Non-panc & 65 & 0.346 & $1.42(0.68-2.92)$ & 0.981 & $0.99(0.38-2.45)$ & 0.086 & $1.99(0.91-4.47)$ \\
\hline \multirow[t]{2}{*}{ PBD } & - & 64 & & 1 & & 1 & & 1 \\
\hline & + & 94 & 0.135 & $1.78(0.84-3.94)$ & 0.004 & $1.61(1.42-21.98)$ & 0.576 & $1.26(0.57-2.93)$ \\
\hline \multirow[t]{2}{*}{ Period of PAA } & P0D2 & 71 & & 1 & & 1 & & 1 \\
\hline & P0D1 & 87 & 0.272 & $0.66(0.31-1.37)$ & 0.380 & $0.66(0.25-1.65)$ & 0.707 & $0.86(0.38-1.89)$ \\
\hline
\end{tabular}

SSI, Surgical Site Infection; BMI, body mass index; DM, diabetes mellitus; Alb, albumin; panc, pancreatic disease; PBD, preoperative biliary drainage; POD, post-operative day; PAA, prophylactic antibiotics administration.

responding institutions. Furthermore, in more than $80 \%$ of the institutions, prophylactic antibiotics were administered for more than four days after PD (including the day of surgery). These results suggested that surgeons believed that long-term administration of antibiotics could lower the incidence of SSIs. However, to date there has been no clinical evidence published showing the optimal duration of prophylactic antibiotics administration for PD.

In this study, we compared mortality, morbidity and infectious complications, including SSI, after PD taking into account the period of prophylactic antibiotics administration. The results of this study indicated that shortening the antibody administration period from 3 to 2 postoperative days did not increase overall complications, infectious complications or SSI. Bacteria detected in patient samples showed a similar distribution in both groups. It seems that these results can be interpreted as indicating that longer use of systemic prophylactic antibiotics did not influence the rate of postoperative infection in patients undergoing PD.

The only predictive pre-operative factor influencing the rate of infectious complications was whether or not the patient underwent PBD, and this was closely related to the development of superficial SSI. Recently, Van der Gaag et al. reported results of a multi-center, randomized trial showing that endoscopic PBD did not have a beneficial effect on surgical outcomes for patients with pancreatic head cancer (18). Furthermore, it has been reported that among patients who underwent PBD, approximately $80 \%$ had a positive bile culture at the time of PD, resulting in infectious complications (19-21). They therefore did not recommend a routine PBD for patients who planned to undergo PD. However, there are inevitably situations in which PBD must be carried out before PD, for example, pre-operative therapy for pancreatic cancer and preoperative evaluation for tumor extension of bile duct cancer. Sudo et al. pointed out that PBD had a notable influence on bile microbial contamination and therefore recommended specific antibiotic prophylaxis for 4 or 5 days post-operatively, based on bile culture results, to prevent infectious complications in pancreatoduodenectomy patients who undergo PBD (22). Routine antibiotic prophylaxis relying on broadspectrum cephalosporins for a long time may be adequate for patients who must undergo PBD.

Nowadays, neoadjuvant chemo(radio)therapy is an advanced treatment option for patients with pancreatic cancer. Actually, some authors have reported favorable survival results at 5 years following surgical resection for pancreatic cancer patients $(23,24)$. Although the issue of PBD is clinically relevant, PBD should be carried out for patients who undergo neoadjuvant chemo(radio)therapy. It was reported that PBD during the period of neoadjuvant treatment might be best achieved with metal stents, which have a higher patency than plastic stents and do not affect the outcome of surgery $(25,26)$. When PBD is necessary, therefore, it should be carried out with metal stents. The present study showed that Enterobacter and Enterococcus species were detected by culture at high frequency in the wounds and in intraabdominal fluid of this study group. Our results are consistent with other reports that Enterococcus species and polymicrobial infections of Gram-negative bacilli commonly develop in bile cultures in patients who undergo PD (27).

We have used $\mathrm{CMZ}$ as a prophylactic antibiotic for 
patients who undergo PD. CMZ is a second generation cephalosporin that is effective in eradicating Enterobacter species, but it has only a weak effect on Enterococcus species, which is a low virulence bacilli. The Guidelines by the Surgical Infection Society and the Infectious Diseases Society of America report that empiric coverage of Enterococcus species is not necessary in patients with community-acquired intraabdominal infection. They describe appropriate antimicrobial regimens for patients with communityacquired intra-abdominal infection of mild-to-moderate severity (Grading the recommendation A-1) (28).

Remedial antibiotics were administered for approximately $30 \%$ of the patients who received prophylactic antibiotics for a limited time period, and carbapenem antibiotics and new quinolone antibiotics were administrated as remedial antibiotics in some of these cases. Recently, the emergence of antibiotic-resistant bacteria has become a problem due to the abuse of broad-spectrum antibiotics. Therefore, it may be necessary to administer third- or fourth generation cephalosporins to stable patients. These antibiotics can cover bacteria identified in the wound or the cultured drained fluids. Depending on the culture results, alternative remedial antibiotics may need to be administered.

The main limitation to this study is the retrospective nature of the analysis, which could have biased the outcome; however, it should be noted that a common staff followed standardized peri-operative management procedures since 2004, as previously reported (12). Actually, there were no significant differences in clinical backgrounds and perioperative management (date to initiate the Per OS, to remove drains, and to discharge from hospital) between the two groups studied.

\section{CONCLUSION}

In conclusion, the administration of prophylactic antibiotics through postoperative day- 1 following PD has been shown to be safe, in conjunction with a policy to immediately administrate remedial antibiotics to patients with clinical sepsis. The duration of prophylactic antibiotics administration did not affect the incidence of SSI. However, prophylactic broad-spectrum antibiotics should be administered over longer periods for patients having high risk factors for SSI.

\section{Conflict of interest statement}

Tomohisa Yamamoto and other co-authors have no conflict of interest.

\section{Disclosure}

All authors declare nothing to disclose.

\section{REFERENCE}

1. Cameron JL, Pitt HA, Yeo CJ, Lillemoe KD, Kaufman HS, Coleman J. One-hundred and forty-fi ve consecutive pancreaticoduodenectomies without mortality. Ann Surg 1993; 217: 430-8.

2. Gouma DJ, van Geenen RC, van Gulik TM, de Haan RJ, de Wit LT, Busch OR,et al. Rates of complications and death after pancreaticoduodenectomy: risk factors ad the impact of hospital volume. Ann Surg 2000;232:786-95.

3. Gouillat C, Gigot JF. Pancreatic surgical complications-the case for prophylaxis. Gut 2001;49:32-9.

4. Lieberman MD, Kilburn H, Lindsey M, Brennan MF. Relation of perioperative deaths to hospital volume among patients undergoing pancreatic resection for malignancy. Ann Surg 1995;222:638-45.

5. Satoi S, Takai S, Matsui Y, Terakawa N, Iwaki R, Fukui J, et al. Less morbidity after pancreaticoduodenectomy of patients with pancreatic cancer. Pancreas 2006;33:45-52.

6. Satoi S, Toyokawa H, Yanagimoto H, Yamamoto T, Yamao J, Kim S, et al. A new guideline to reduce postoperative morbidity after pancreaticoduodenectomy. Pancreas. 2008 Aug;37(2):128-33.

7. Kaiser $A B$. Antimicrobial prophylaxis in surgery. $N$ Engl $J$ Med 1986;315:1129-38.

8. Targarona EM. Single-does antibiotic prophylaxis in patients at high risk for infection in biliary surgery: a prospective and randomized study comparing cefonicid with mezlocillin. Surgery 1990;107: 327-34.

9. Mangram AJ. Guidelines for prevention of surgical site infection, 1999. Hospital Infection Control Practices Advisory Committee. Infect Control Hosp Epidemiol 1999;20:250-78; quiz, 279-80.

10. Imamura H, Kurokawa Y, Tsujinaka T, Inoue K, Kimura Y, lijima S, et al. Intraoperative versus extended antimicrobial prophylaxis after gastric cancer surgery: a phase 3, open-label, randomised controlled, noninferiority trial. Lancet Infect Dis. 2012 May;12(5):381-7.

11. Satoi S, Toyokawa H, Yanagimoto H, Yamamoto T, Hirooka S, Yui R, et al. Is a nonstented duct-to-mucosa anastomosis using the modified Kakita method a safe procedure? Pancreas. 2010 Mar;39(2):165-70.

12. Yamaki S, Satoi S, Toyokawa H, Yanagimoto H, Yamamoto T, Hirooka $\mathrm{S}$, et al. The clinical role of critical pathway implementation for pancreaticoduodenectomy in 179 patients. J Hepatobiliary Pancreat Sci. 2012 Mar 10.

13. American College of Chest Physicians/Society of Critical Care Medicine Consensus Conference: definitions for sepsis and organ failure and guidelines for the use of innovative therapies in sepsis. Crit Care Med 1992;20:864-74.

14. Bassi C, Dervenis C, Butturini G, Fingerhut A, Yeo C, Izbicki J, et al. Postoperative pancreatic fistula: an international study group (ISGPF) definition. Surgery. 2005;138:8-13.

15. Wente MN, Bassi C, Dervenis C, Fingerhut A, Gouma DJ, Izbicki JR, et al. Delayed gastric emptying (DGE) after pancreatic surgery: a suggested definition by the International Study Group of Pancreatic Surgery (ISGPS). Surgery. 2007 Nov;142(5):761-8.

16. Obesity: Preventing and managing the global epidemic; Report of a WHO Consultation on Obesity, Geneva, 3-5, June 1997. Geneva: WHO; 1998.

17. Yoshinobu Sumiyama, Shinya Kusachi, Yuichi Yoshida. Questionnaire on Perioperative Antibiotic Therapy in 2003:Postoperative Prophylaxis. Surg Today (2006) 36:107-113

18. van der Gaag NA, Rauws EA, van Eijck $\mathrm{CH}$, Bruno MJ, van der Harst $\mathrm{E}$, Kubben FJ, et al. Preoperative biliary drainage for cancer of the head of the pancreas. N Engl J Med. 2010 Jan 14;362(2):129-37.

19. Mezhir JJ, Brennan MF, Baser RE, D'Angelica MI, Fong Y, DeMatteo $\mathrm{RP}$, at al. A matched case-control study of preoperative biliary drainage in patients with pancreatic adenocarcinoma: routine drainage is not justified. J Gastrointet Surg. 2009;13:2163-2169.

20. Coates JM, Beal SH, Russo JE, Vanderveen KA, Chen SL, Bold RJ, et 
al. Negligible effect of selective preoperative biliary drainage on perioperative resuscitation, morbidity, and mortality in patients undergoing pancreaticoduodenectomy. Arch Surg. 2009;144:841-847.

21. Lermite E, Pessaux P, Teyssedou C, Etienne S, Brehant O, Arnaud JP. Effect of preoperative endoscopic biliary drainage on infectious morbidity after pancreatoduodenectomy: a case-control study. Am J Surg. 2008;195:442-446.

22. Sudo T, Murakami Y, Uemura K, Hayashidani $Y$, Hashimoto $Y$, Ohge $H$, et al. Specific antibiotic prophylaxis based on bile cultures is required to prevent postoperative infectious complications in pancreatoduodenectomy patients who have undergone preoperative biliary drainage. World J Surg. 2007;31:2230-2235.

23. Katz MH, Wang H, Fleming JB, Sun CC, Hwang RF, Wolff RA, et al. Long-term survival after multidisciplinary management of resected pancreatic adenocarcinoma. Ann Surg Oncol. 2009;16:836-47.

24. Satoi S, Yanagimoto H, Toyokawa H, Yamamoto T, Hirooka S, Yui R, et al. Long-term results of surgical resection after preoperative chemoradiation in patients with pancreatic cancer. Pancreas. 2012;41(2):333-5

25. Davids PH, Groen AK, Rauws EA, Tytgat GN, Huibregtse K. Randomised trial of self-expanding metal stents versus polyethylene stents for distal malignant biliary obstruction. Lancet 1992;340:1488-92.

26. Mullen JT, Lee JH, Gomez HF, Ross WA, Fukami N, Wolff RA, et al. Pancreaticoduodenectomy after placement of endobiliary metal stents. J Gastrointest Surg 2005;9:1094-104.

27. Povoski SP, Karpeh MS Jr, Conlon KC, Blumgart LH, Brennan MF. Preoperative biliary drainage: impact on intraoperative bile cultures and infectious morbidity and mortality after pancreaticoduodenectomy. J Gastrointest Surg. 1999 Sep-0ct; 3(5):496-505.

28. Solomkin JS, Mazuski JE, Bradley JS, Rodvold KA, Goldstein EJ, Baron EJ, et al. Diagnosis and management of complicated intraabdominal infection in adults and children: guidelines by the Surgical Infection Society and the Infectious Diseases Society of America. Clin Infect Dis. 2010 Jan 15;50(2):133-64. 